\title{
Molecular Modeling and Molecular Dynamics Simulation Studies on the Selective Binding Mechanism of MTHFD2 Inhibitors
}

\author{
Mengyang $Q u^{1,2}$ \\ ${ }^{1}$ Shanghai Institute of Applied Physics, Chinese Academy of Sciences, Shanghai, China \\ ${ }^{2}$ University of Chinese Academy of Sciences, Beijing, China \\ Email: qumengyang@sinap.ac.cn
}

How to cite this paper: Qu, M.Y. (2022) Molecular Modeling and Molecular Dynamics Simulation Studies on the Selective Binding Mechanism of MTHFD2 Inhibitors. Computational Molecular Bioscience, 12, 1-11.

https://doi.org/10.4236/cmb.2022.121001

Received: December 7, 2021

Accepted: March 4, 2022

Published: March 7, 2022

Copyright ( 2022 by author(s) and Scientific Research Publishing Inc. This work is licensed under the Creative Commons Attribution International License (CC BY 4.0).

http://creativecommons.org/licenses/by/4.0/ (c) (i) Open Access

\begin{abstract}
Methylenetetrahydrofolate dehydrogenase 2 (MTHFD2) is a mitochondrial enzyme that plays an important role in purinecarbon metabolism and thymidine biosynthesis. It has attracted broad interest as a novel therapeutic target for cancer. However, a major problem of current MTHFD2 inhibitors is their lack of selectivity and reactivity with its closest isoform, MTHFD1. Recently, the first selective MTHFD2 inhibitor, DS44960156, has been reported and it exhibits a more than 18-fold selectivity for MTHFD2 over MTHFD1. However, mechanism of DS44960156 selective binding to MTHFD2 over MTHFD1 is unknown. In this study, molecular docking, molecular dynamics (MD) simulations, molecular mechanics generalized born/surface area (MM_GBSA) binding free energy calculations, and analysis of the decomposition of binding free energies were used to investigate the selective binding mechanism of DS44960156 to the folate-binding site of MTHFD2 over MTHFD1. The results revealed that contributions from residues Gln100/Gln132, Val55/Asn87, and Gly237/Gly310 in the binding pocket of MTHFD1/MTHFD2 are the key factors responsible for the binding selectivity. These findings explain the selectivity of DS44960156 to MTHFD2 over MTHFD1, and may provide guidance for the future study and design of novel MTHFD2 inhibitors.
\end{abstract}

\section{Keywords}

Methylenetetrahydrofolate Dehydrogenase 2, Molecular Docking, Molecular Dynamics Simulations

\section{Introduction}

Anticancer drugs such as alkylating agents, topoisomerase inhibitors, and anti-microtubule agents directly target DNA replication and cell division; however, 
they affect normal cells as well as cancer cells. Methylenetetrahydrofolate dehydrogenase 2 (MTHFD2) as a novel therapeutic target for cancer has attracted broad interest [1]. MTHFD2 is a mitochondrial enzyme that plays an important role in purine carbon metabolism and thymidine biosynthesis. Sex-determining region Y-related high mobility group box 7, SOX7, as a tumor suppressor, can combine with the MTHFD2 promoter to inhibit the expression of MTHFD2. Therefore, MTHFD2 is not expressed in most healthy adults [2]. However, the induction of KRAS can activate the AKT and ERK1/2 pathways, and recognize and bind to the MTHFD2 promoter through c-Myc to up-regulate the expression of MTHFD2. High expression of MTHFD2 mRNA and protein has been found in patients with various types of cancer [3] [4] [5]. Thus, MTHFD2 inhibitors are considered as potential cancer treatment drugs with minimal side effects. A few of MTHFD2 inhibitors, such as LY345899 [6] [7] and carolacton [8] have been identified. However, an important problem of these inhibitors is the lack of selectivity for MTHFD2 over MTHFD1, which is because of the similar structure of the 2 enzymes. As shown in Figure 1, MTHFD1 shares 80\% similarity with MTHFD2 in the folate-binding site. Inhibition of MTHFD1 is considered to be a potential safety risk, because it is broadly expressed in normal tissues [9]. Therefore, selective inhibitors of MTHFD2 are highly attractive for cancer treatment and drug development.

Recently, Kawai et al. reported the first selective inhibitor of MTHFD2, DS44960156 (Figure 2(a)), which exhibits a more than 18-fold selectivity for MTHFD2 over MTHFD1 [9]. X-ray analysis of the crystal structure of the MTHFD2-DS44960156 complex shows that DS44960156 occupies the folate-binding site of MTHFD2 (Figure 2(b)). The key interactions of the MTHFD2-DS44960156 complex are: 1) Four hydrogen bonds: Gln132 and Lys88

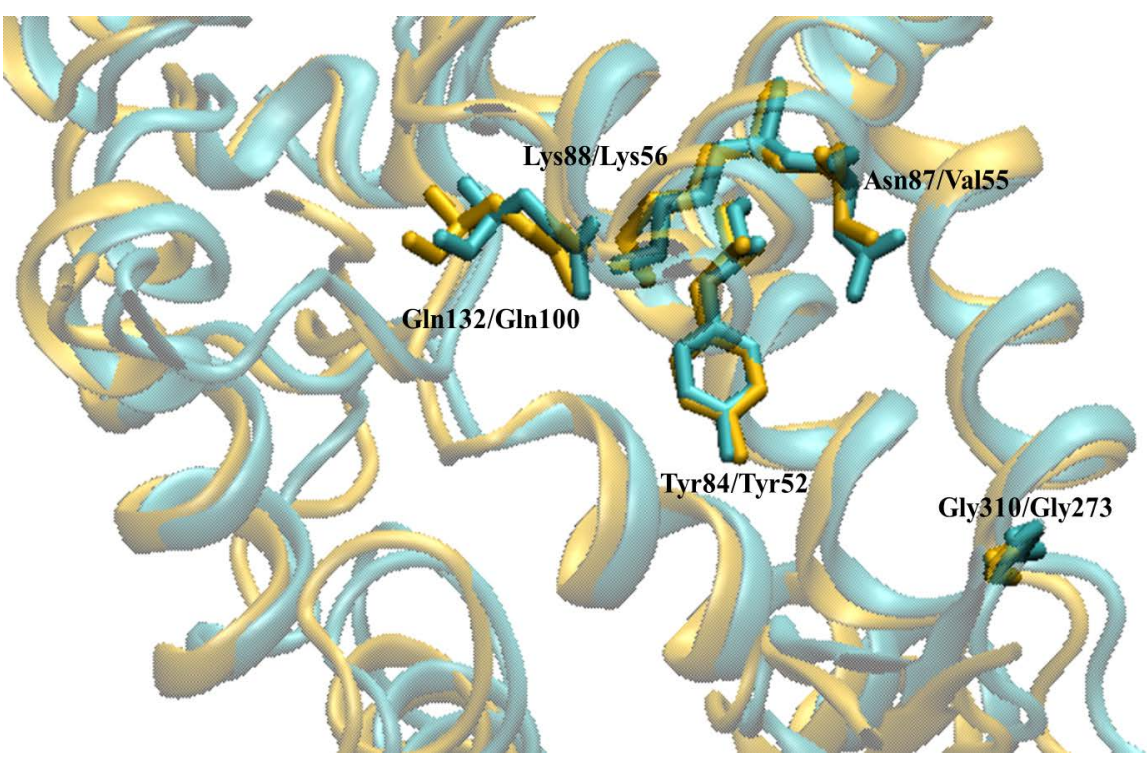

Figure 1. Structural alignment of the folate-binding site of MTHFD1 (orange) and MTHFD2 (cyan). 
(a)<smiles>O=C(O)c1ccc(C(=O)N2CCc3c(c(=O)oc4ccccc34)C2)cc1</smiles>

(b)

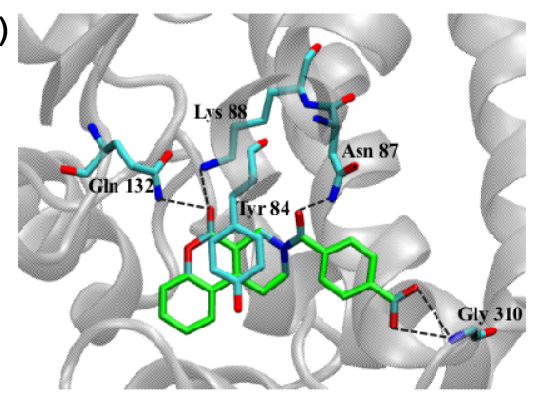

Figure 2. (a) Chemical structure of DS44960156. (b) The binding pocket of DS44960156 in MTHFD2.

in MTHFD2 with $\mathrm{C}=\mathrm{O}$ of the pyrimidin-4-one in DS44960156; Asn87 in MTHFD2 with $\mathrm{C}=\mathrm{O}$ of the linker amide in DS44960156; Gly310 in MTHFD2 with $S=O$ of the sultam in DS44960156; 2) Anda $\pi-\pi$ interaction between Tyr84 in MTHFD2 and the pyrimidin-4-one in DS44960156. However, because of the highly similar structures between the folate-binding sites of MTHFD1 and MTHFD2, it is unclear why DS44960156 selectively binds to MTHFD2 over MTHFD1.

In this study, molecular modeling and molecular dynamics (MD) simulations were performed to investigate the selective binding mechanism of DS44960156 to MTHFD2 over MTHFD1. We performed MD simulations on the complex structures of DS44960156-MTHFD1 and DS44960156-MTHFD2. The conformation of DS44960156 in the folate-binding site of MTHFD1 and MTHFD2 was compared. Binding free energies were calculated to identify the key residues responsible for the different binding affinity. These results illustrate the selectivity of DS44960156, and may provide guidance for design of novel MTHFD2 inhibitors.

\section{Material and Methods}

\subsection{System Preparations}

The crystal structure of MTHFD2-DS44960156 (PDB code 6JIB) [9] was downloaded from the RCSB Protein Data Bank, and the structure of MTHFD1DS44960156 was predicted by molecular docking. Docking of DS44960156 to the folate-binding site of MTHFD1 was performed using the AutoDock Vina package [10], and the crystal structure of MTHFD1-LY345899 (PDB code 6ECQ) [11] with LY345899 deleted was used for the docking. All non-bonded hetero-atoms and water molecules were removed fromMTHFD1, polar hydrogen atoms and subsequently the Kollman united atom partial charges were added and assigned to MTHFD1. All hydrogen atoms, including polar and nonpolar atoms, and the Gasteiger-Hückel atomic charge were added and assigned to DS44960156. The MTHFD1 receptor was treated as rigid, and DS44960156 was treated as flexible during docking. We created a $40 \times 40 \times 40 \AA^{3}$ rectangular parallelepiped around the cavity, ensuring that it is the right size to allow the ligand to rotate in it. The best pose of the complex, according to the criteria of lowest energy, was selected 
for MD simulation steps.

\subsection{Simulations}

MD simulations of the complex structures of DS44960156 with MTHFD2 and MTHFD1 were performed. The general AMBER force field (GAFF) [12] was used to parameterize the inhibitor DS44960156 and NADP. The electrostatic potential of DS44960156 was calculated at the $\mathrm{HF} / 6-31 \mathrm{G}^{*}$ level using the program Gaussian 09. Afterward, the missing force field parameters were generated using the antechamber module in AMBER 20 [13]. The AMBER ff14SB force field was used to parameterize MTHFD1 and MTHFD2. The complexes were solvated in TIP3P [14] water boxes with an internal offset distance of $10.0 \AA$ A. Calcium and chlorine ions were added to neutralize the system.

All of the MD simulations were performed using GROMACS-5.0.7 software [15]. Firstly, the systems were subjected to energy minimizations in 2 steps [16]. The solvent water molecules were optimized first, while the coordinates of other molecules were fixed, followed by an unrestrained minimization of all the atoms in the system. Secondly, the system was gradually heated to $300 \mathrm{~K}$ under constant volume. Thirdly, a $5 \mathrm{~ns}$ simulation under constant volume and temperature was performed with the non-hydrogen atoms of the protein fixed, followed by a 5 ns simulation under constant pressure and temperature. Finally, 300 ns production simulation was performed. The temperature was maintained at $300 \mathrm{~K}$ with a coupling coefficient of 1.0 ps through the V-rescale method. The particle-mesh Ewald method [17] was used to treat long-range electrostatic interactions with a real space cutoff of $1 \mathrm{~nm}$ and the cutoff of the van der Waals interactions was set to $1 \mathrm{~nm}$. Bonds involving hydrogen atoms were constrained using the LINCS algorithm [18]. The time step was set to $2 \mathrm{fs}$ and the periodic boundary conditions were applied in all directions.

\subsection{MM/GBSA Free Energy Calculations and Decomposition}

The binding free energy ( $\Delta G_{\text {bind }}$ ) between DS44960156 and MTHFD1/2 was calculated by the MM/GBSA method: [19] [20] [21] [22]

$$
\begin{aligned}
\Delta G_{\text {bind }} & =G_{\text {complex }}-\left(G_{\text {receptor }}+G_{\text {ligand }}\right) \\
& =\Delta E_{M M}+\Delta E_{\text {solvation }}-T \Delta S \\
& =\Delta E_{M M}+\Delta G_{G A}+\Delta G_{S B}-T \Delta S
\end{aligned}
$$

where $G_{\text {complex }}, G_{\text {receptor }}$, and $G_{\text {ligand }}$ are the free energies of the complex, receptor, and ligand, respectively; $\Delta E_{M M}$ is the gas-phase interaction energy between the receptor and the ligand; $\Delta G_{G A}$ and $\Delta G_{S B}$ are respectively the non-polar and polar parts of the desolvation free energy $\Delta E_{\text {solvation }} . \Delta G_{S A}$ is calculated based on the solvent-accessible surface area (SASA): $\Delta G_{S A}=0.0072 \Delta$ SASA [23]. And $\Delta G_{G B}$ is calculated using the GB model [24]. The binding free energies were then decomposed into contributions of protein-ligand interaction pairs using 
the MM_GBSA method. The conformational entropy $(-T \Delta S)$ was calculated using the method provided by Duan et al. [25] [26]. In the method, the interaction entropy can be calculated by the following formula:

$$
-T \Delta S=k T \ln \left\langle\mathrm{e}^{\beta \Delta E_{i n t}^{p l}}\right\rangle,
$$

where $\Delta E_{\text {int }}^{p l}=E_{i n t}^{p l}-\left\langle E_{i n t}^{p l}\right\rangle$, represents the fluctuation of protein-ligand interaction energy around the average energy, and $\langle\ldots\rangle$ stands for a statistical average.

\section{Results and Discussion}

\subsection{Molecular Docking}

In this study, the complex structure of MTHFD1-DS44960156 was predicted by molecular docking. To examine the prediction accuracy, we first performed re-docking of DS44960156 into MTHFD2. The re-docked conformation was then superimposed onto the crystal conformation (Figure 3(a)). The rootmean-square deviation (RMSD) of the heavy atoms of DS44960156 between the redocked conformation and the crystal conformation was $0.4 \AA$, suggesting that the crystal structure of the receptor-ligand complex could be accurately reproduced by AutoDock Vina. The docking results of MTHFD1-DS44960156 (Figure 3(b)) showed that DS44960156 mainly adopts 1 conformation in the folate-binding site of MTHFD1. AutoDock Vina calculates the binding free energy between the receptor and each docked ligand for each pose, and the pose with the lowest binding free energy was used for the following MD study.

\subsection{Structural Stability}

MD simulations can accurately identify correct binding conformations, and provide valuable information of the dynamic properties of receptor-ligand interactions [27]. We then performed $300 \mathrm{~ns} \mathrm{MD}$ simulations on the crystal structure of MTHFD2-DS44960156 and the docked structure of MTHFD1-DS44960156. The RMSD for all the $C_{\alpha}$ atoms of the 2 proteins relative to the initial structure was
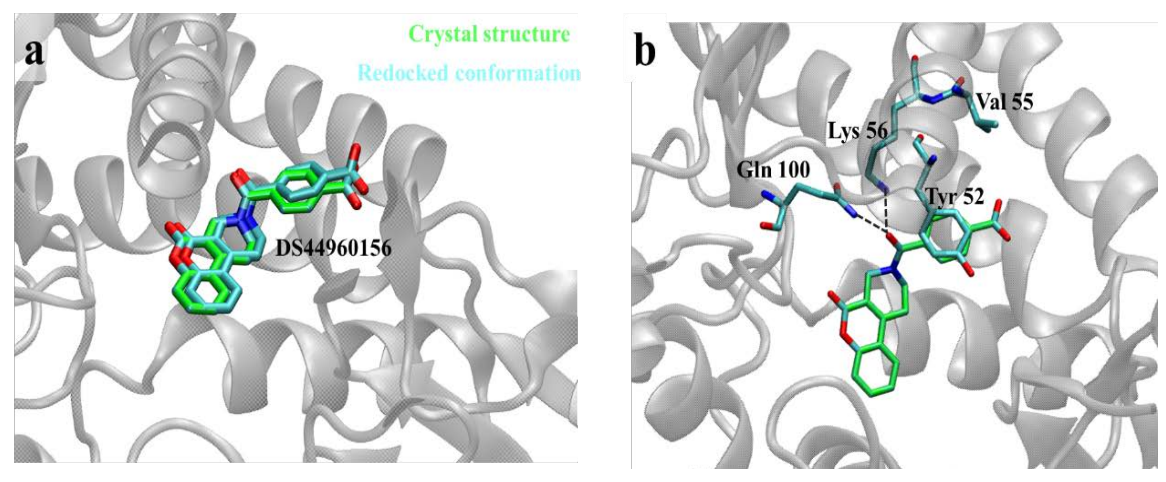

Figure 3. (a) Superimposition of the crystal conformation (carbon atoms of DS44960156 colored in green) and re-docked conformation (carbon atoms of DS44960156 colored in cyan) of the MTHFD2-DS44960156 complex. (b) The docked conformation of the MTHFD1-DS44960156 complex. 
calculated. As shown in Figure 4(a), both systems reached stability from the beginning of the simulations. To further understand the stability of DS44960156 in the folate-binding sites of MTHFD2 and MTHFD1, the RMSD of heavy atoms of DS44960156 was calculated. As shown in Figure 4(b), DS44960156 exhibited significantly larger conformational dynamics in MTHFD1 than in MTHFD2. These results indicate that DS44960156 is more stable in the folate-binding site of MTHFD2 which may be due to its high binding affinity.

The difference of the RMSDs between DS44960156 in the folate-binding sites of MTHFD1 and MTHFD2 indicates that DS44960156 may have a different binding mode in MTHFD1 and MTHFD2. To further understand this finding, we superimposed the frames of the MD simulations of MTHFD1-DS44960156 and MTHFD2-DS44960156. As shown in Figure 5, DS44960156 occupied the folate-binding site ofMTHFD1 as well as MTHFD2, with the terminal benzene ring points out of the pocket, and carboxyl group points into the pocket. However, DS44960156 in MTHFD1 is farther from the folate-binding site than in MTHFD2, which may cause the different binding affinity, thus the difference of the RMSDs.

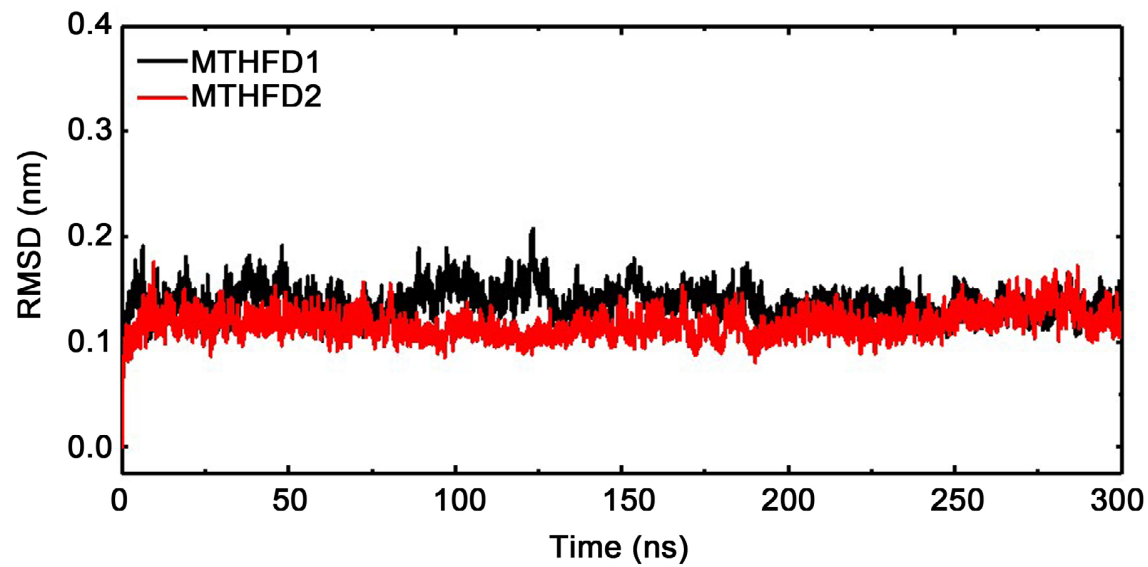

(a)

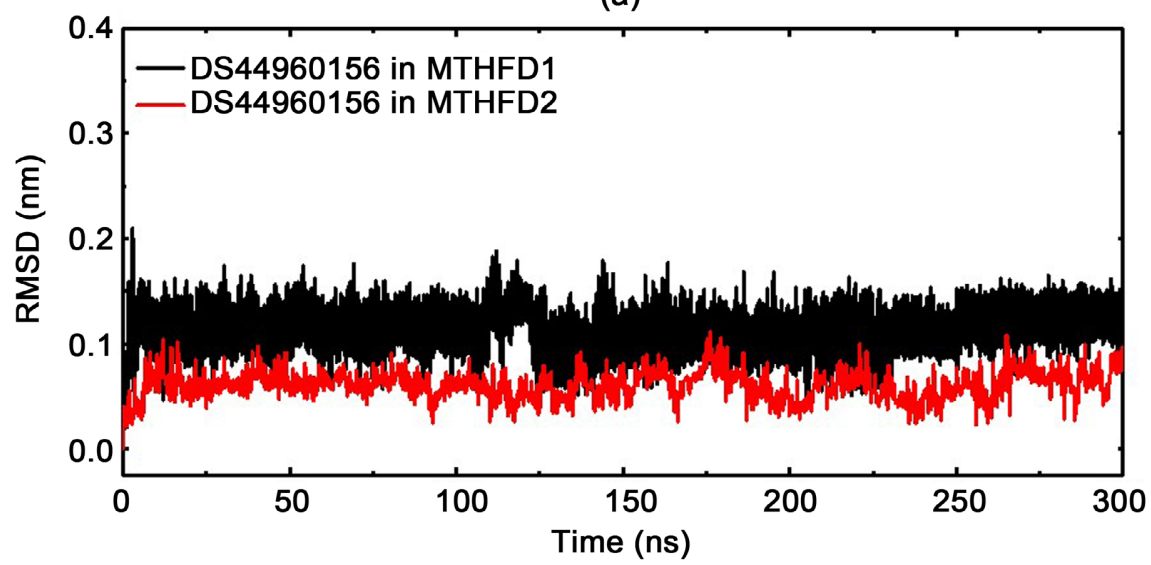

(b)

Figure 4. (a) The RMSDs for all the $C_{\alpha}$ atoms of MTHFD1 and MTHFD2 during the MD simulations. (b) The RMSDs for the heavy atoms of DS44960156 in the folate-binding sites of MTHFD1 and MTHFD2 during the MD simulations. 


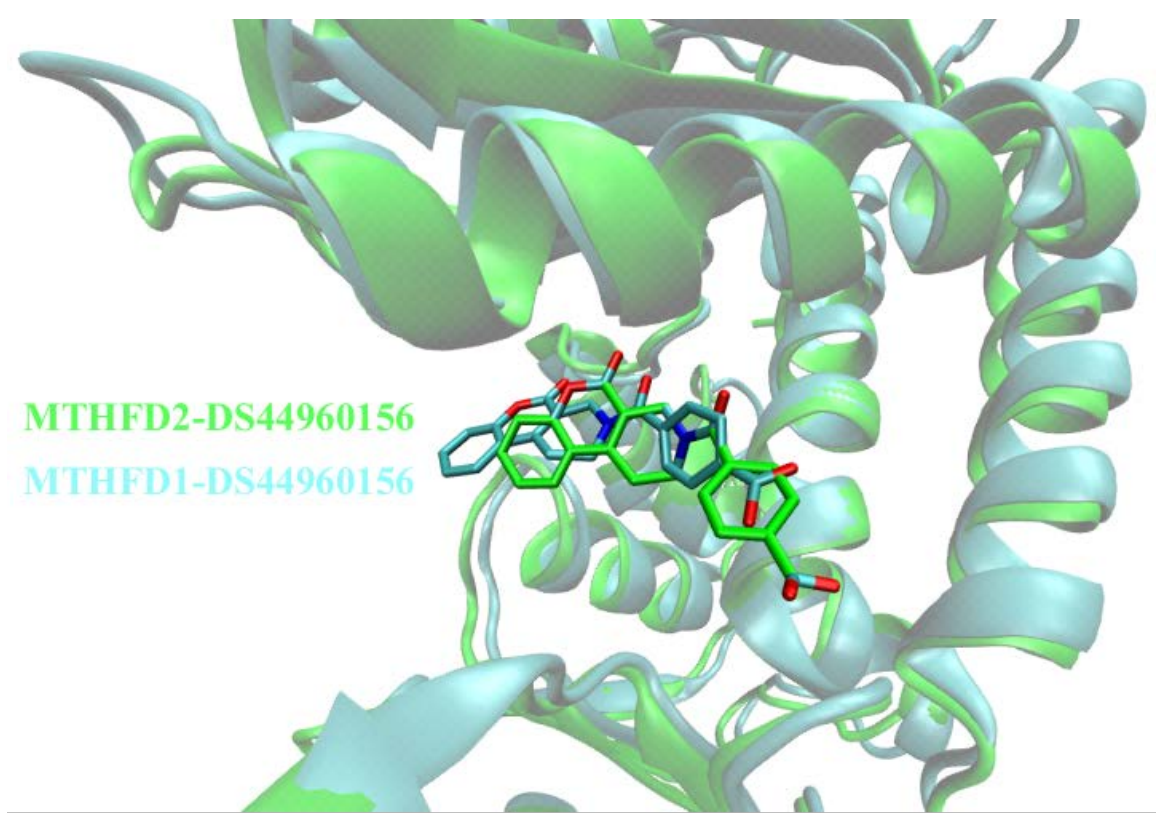

Figure 5. Superimposition of frames from the MD simulations of MTHFD2-DS44960156 and MTHFD1-DS44960156.

\subsection{Binding Free Energy}

To further understand the binding affinity between DS44960156 and MTHFD1/2, MM_GBSA binding free energy calculations were performed on the last $50 \mathrm{~ns}$ MD trajectories. As shown in Table 1, both the van der Waals $\left(\Delta E_{v d w}\right)$ and electrostatic $\left(\Delta E_{\text {ele }}\right)$ energy are dominant contributions to the binding affinity in each system, while the $\Delta E_{v d w}$ values are much larger than the $\Delta E_{\text {ele }}$ values, indicating the importance of hydrophobic interactions in both systems. The calculated binding free energy $\left(\Delta G_{b i n d}\right.$ ) of MTHFD1-DS44960156 was $52.7 \mathrm{~kJ} / \mathrm{mol}$ lower than that of MTHFD2-DS44960156. The reduction was mainly due to $\Delta E_{v d w}$ and $\Delta E_{\text {ele }}$, indicating that both the hydrophobic and electrostatic interactions are unfavorable in the MTHFD1-DS44960156 system compared with the MTHFD2-DS44960156 system.

\subsection{Residue-Specific Binding Free Energies}

In order to explore the reasons for the stronger binding affinity to MTHFD2, the decomposition of $\Delta G_{b i n d}$ for the MTHFD1-DS44960156 and MTHFD2DS44960156 systems was performed using the MM_GBSA method. Residues that contributed more than $5 \mathrm{~kJ} / \mathrm{mol}$ were defined as hot-spot residues. As shown in Table 2, all of the residues in the binding pocket of MTHFD2 were hot-spot residues. However, there were only 2 hot-spot residues (Tyr 52 and Lys 56) in MTHFD1. These results indicate that the contributions from residues Gln100/Gln132, Val55/Asn87, and Gly237/Gly310 in the binding pockets of MTHFD1/2 are the key factors responsible for the binding selectivity of DS44960156.

The positions of these residues in the 2 complexes are shown in Figure 6. It 
Table 1. Binding free energy (kJ/mol) between DS44960156 and MTHFD1 and MTHFD2.

\begin{tabular}{ccc}
\hline $\begin{array}{c}\text { Energy items } \\
(\mathrm{kJ} / \mathrm{mol})\end{array}$ & Complex & \\
\hline & MTHFD1-DS44960156 & MTHFD2-DS44960156 \\
$\Delta E_{v d w}$ & $-125.900 \pm 7.135$ & $-147.832 \pm 5.567$ \\
$\Delta E_{\text {ele }}$ & $-31.892 \pm 5.426$ & $-62.591 \pm 4.586$ \\
$\Delta G_{G B}$ & $101.347 \pm 13.105$ & $110.361 \pm 4.637$ \\
$\Delta G_{S A}$ & $-14.342 \pm 0.711$ & $-19.305 \pm 0.802$ \\
$-T \Delta S$ & $25.342 \pm 2.353$ & $20.798 \pm 3.653$ \\
$\Delta G_{\text {bind }}$ & $-39.646 \pm 9.842$ & $-92.396 \pm 6.892$ \\
\hline
\end{tabular}

Table 2. Energy contributions $(\mathrm{kJ} / \mathrm{mol})$ of residues in the binding pocket of MTHFD1 and MTHFD2.

\begin{tabular}{cccc}
\hline Residues of MTHFD1 & Binding energy & Residues of MTHFD2 & Binding energy \\
\hline Tyr 52 & $-6.062 \mathrm{~kJ} / \mathrm{mol}$ & Tyr 84 & $-13.201 \mathrm{~kJ} / \mathrm{mol}$ \\
Val 55 & $-1.372 \mathrm{~kJ} / \mathrm{mol}$ & Asn 87 & $-8.763 \mathrm{~kJ} / \mathrm{mol}$ \\
Lys 56 & $-9.103 \mathrm{~kJ} / \mathrm{mol}$ & Lys 88 & $-9.134 \mathrm{~kJ} / \mathrm{mol}$ \\
Gln 100 & $-3.361 \mathrm{~kJ} / \mathrm{mol}$ & Gln 132 & $-7.046 \mathrm{~kJ} / \mathrm{mol}$ \\
Gly 273 & $-0.442 \mathrm{~kJ} / \mathrm{mol}$ & Gly 310 & $-6.105 \mathrm{~kJ} / \mathrm{mol}$ \\
\hline
\end{tabular}
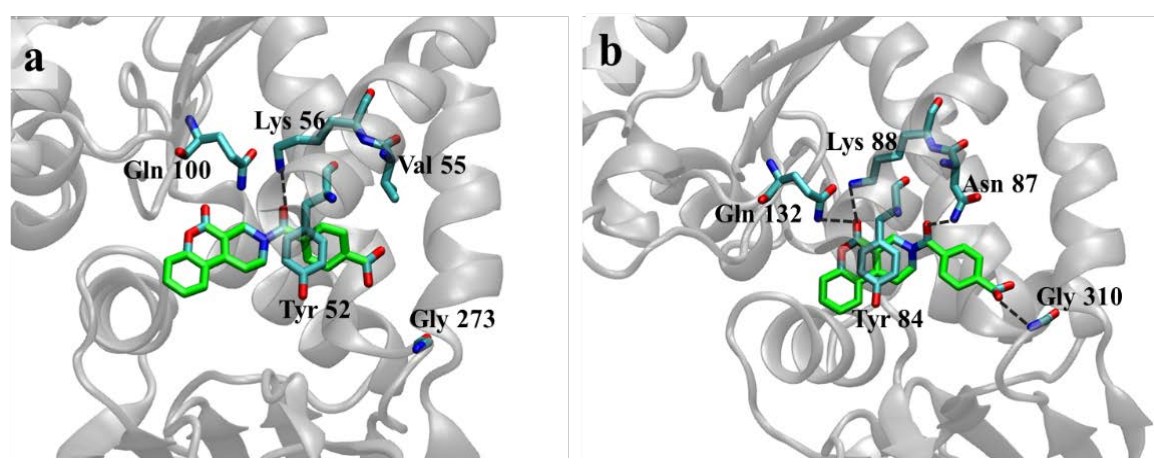

Figure 6. Snapshots of the residue interaction with DS44960156 in the MD simulations of (a) MTHFD1 and (b) MTHFD2.

can be seen that MTHFD2 forms 4 hydrogen bonds (Gln132/Lys88 with $\mathrm{C}=\mathrm{O}$ of the pyrimidin-4-one; Asn87 with $\mathrm{C}=\mathrm{O}$ of the linker amide; $\mathrm{Gly} 310$ with $\mathrm{S}=\mathrm{O}$ of the sultam), and a strong $\pi-\pi$ interaction (Tyr84 and pyrimidin-4-one) with DS44960156. However, in the MTHFD1-DS44960156 complex, DS44960156 only forms a hydrogen bond and a weak $\pi-\pi$ interaction with Lys56 and Tyr52 in MTHFD1. The potential reasons for these interaction differences may be that residue Asn 87 is replaced by a non-polar residue (Val 55) in MTHFD1 which cannot interact with the $\mathrm{C}=\mathrm{O}$ of the linker amide in DS44960156. This causes the position of DS44960156 in MTHFD1 to be farther from the folate-binding site than in MTHFD2. Therefore, the $\mathrm{C}=\mathrm{O}$ of pyrimidin-4-one in DS44960156 
cannot interact with MTHFD1, and the $\pi-\pi$ interaction between Tyr84 and DS44960156 is weak. These interaction differences may be responsible for the difference in selectivity.

\section{Conclusion}

In this study, molecular docking, MD simulations, MM_GBSA binding free energy calculations, and calculations of the decomposition of binding free energies were performed to explore the selective binding mechanism of DS44960156 to the folate-binding site of MTHFD2 over that of MTHFD1. MM_GBSA binding free energy calculations showed that $\Delta G_{\text {bind }}$ was $52.7 \mathrm{~kJ} / \mathrm{mol}$ lower in the MTHFD1-DS44960156 complex compared with the MTHFD2-DS44960156 complex. The per-residue free energy decomposition shows that all the residues in the binding pocket of MTHFD2 have strong interactions with DS44960156. Nonetheless, the interactions between residues Gln100, Val55 and Gly237 in the binding pocket of MTHFD1 and DS44960156 are quite weak. These results indicate that the contributions from residues Gln100/Gln132, Val55/Asn87, and Gly237/ Gly310 in the folate binding pockets of MTHFD1 and MTHFD2 are the key factors responsible for the binding selectivity of DS44960156. These results might be useful for designing novel selective MTHFD2 inhibitors.

\section{Conflicts of Interest}

The author declares no conflicts of interest regarding the publication of this paper.

\section{References}

[1] Ducker, G.S., Chen, L., Morscher, R.J., Ghergurovich, J.M., Esposito, M., Teng, X., Kang, Y. and Rabinowitz, J.D. (2016) Reversal of Cytosolic One-Carbon Flux Compensates for Loss of the Mitochondrial Folate Pathway. Cell Metabolism, 23, 1140-1153. https://doi.org/10.1016/j.cmet.2016.04.016

[2] Tedeschi, P.M., Vazquez, A., Kerrigan, J.E. and Bertino, J.R. (2015) Mitochondrial Methylenetetrahydrofolate Dehydrogenase (MTHFD2) Overexpression Is Associated with Tumor Cell Proliferation and Is a Novel Target for Drug Development. Molecular Cancer Research, 13, 1361-1366. https://doi.org/10.1158/1541-7786.MCR-15-0117

[3] Nilsson, R., Jain, M., Madhusudhan, N., Sheppard, N.G., Strittmatter, L., Kampf, C., Huang, J., Asplund, A. and Mootha, V.K. (2014) Metabolic Enzyme Expression Highlights a Key Role for MTHFD2 and the Mitochondrial Folate Pathway in Cancer. Nature Communications, 5, Article No. 3128. https://doi.org/10.1038/ncomms4128

[4] Liu, F., Liu, Y., He, C., Tao, L., He, X., Song, H. and Zhang, G. (2014) Increased MTHFD2 Expression Is Associated with Poor Prognosis in Breast Cancer. Tumor Biology, 35, 8685-8690. https://doi.org/10.1007/s13277-014-2111-X

[5] Ju, H.-Q., Lu, Y.-X., Chen, D.-L., Zuo, Z.-X., Liu, Z.-X., Wu, Q.-N., Mo, H.-Y., Wang, Z.-X., Wang, D.-S. and Pu, H.-Y. (2019) Modulation of Redox Homeostasis by Inhibition of MTHFD2 in Colorectal Cancer: Mechanisms and Therapeutic Implications. JNCI: Journal of the National Cancer Institute, 111, 584-596. 
https://doi.org/10.1093/jnci/djy160

[6] Schmidt, A., Wu, H., MacKenzie, R.E., Chen, V.J., Bewly, J.R., Ray, J.E., Toth, J.E. and Cygler, M. (2000) Structures of Three Inhibitor Complexes Provide Insight into the Reaction Mechanism of the Human Methylenetetrahydrofolate Dehydrogenase/Cyclohydrolase. Biochemistry, 39, 6325-6335.

https://doi.org/10.1021/bi992734y

[7] Gustafsson, R., Jemth, A.-S., Gustafsson, N.M., Färnegårdh, K., Loseva, O., Wiita, E., Bonagas, N., Dahllund, L., Llona-Minguez, S. and Häggblad, M. (2017) Crystal Structure of the Emerging Cancer Target MTHFD2 in Complex with a SubstrateBased Inhibitor. Cancer Research, 77, 937-948. https://doi.org/10.1158/0008-5472.CAN-16-1476

[8] Fu, C., Sikandar, A., Donner, J., Zaburannyi, N., Herrmann, J., Reck, M., Wagner-Döbler, I., Koehnke, J. and Müller, R. (2017) The Natural Product Carolacton Inhibits Folate-Dependent C1 Metabolism by Targeting FolD/MTHFD. Nature Communications, 8, Article No. 1529. https://doi.org/10.1038/s41467-017-01671-5

[9] Kawai, J., Ota, M., Ohki, H., Toki, T., Suzuki, M., Shimada, T., Matsui, S., Inoue, H., Sugihara, C. and Matsuhashi, N. (2019) Structure-Based Design and Synthesis of an Isozyme-Selective MTHFD2 Inhibitor with a Tricyclic Coumarin Scaffold. ACS Medicinal Chemistry Letters, 10, 893-898. https://doi.org/10.1021/acsmedchemlett.9b00069

[10] Trott, O. and Olson, A.J. (2010) AutoDock Vina: Improving the Speed and Accuracy of Docking with a New scoring Function, Efficient Optimization, and Multithreading. Journal of Computational Chemistry, 31, 455-461. https://doi.org/10.1002/jcc.21334

[11] Bueno, R., Dawson, A. and Hunter, W.N. (2019) An Assessment of Three Human Methylenetetrahydrofolate Dehydrogenase/Cyclohydrolase-Ligand Complexes Following Further Refinement. Acta Crystallographica Section F: Structural Biology Communications, 75, 148-152. https://doi.org/10.1107/S2053230X18018083

[12] Wang, J., Wolf, R.M., Caldwell, J.W., Kollman, P.A. and Case, D.A. (2004) Development and Testing of a General Amber Force Field. Journal of Computational Chemistry, 25, 1157-1174. https://doi.org/10.1002/jcc.20035

[13] Salomon-Ferrer, R., Case, D.A. and Walker, R.C. (2013) An Overview of the Amber Biomolecular Simulation Package. WIREs Computational Molecular Science, 3, 198-210. https://doi.org/10.1002/wcms.1121

[14] Lamoureux, G., Harder, E., Vorobyov, I.V., Roux, B. and MacKerell Jr., A.D. (2006) A Polarizable Model of Water for Molecular Dynamics Simulations of Biomolecules. Chemical Physics Letters, 418, 245-249. https://doi.org/10.1016/j.cplett.2005.10.135

[15] Abraham, M.J., Murtola, T., Schulz, R., Páll, S., Smith, J.C., Hess, B. and Lindahl, E. (2015) GROMACS: High Performance Molecular Simulations through Multi-Level Parallelism from Laptops to Supercomputers. SoftwareX, 1-2, 19-25. https://doi.org/10.1016/j.softx.2015.06.001

[16] Lu, S., Banerjee, A., Jang, H., Zhang, J., Gaponenko, V. and Nussinov, R. (2015) GTP Binding and Oncogenic Mutations May Attenuate Hypervariable Region (HVR)-Catalytic Domain Interactions in Small GTPase K-Ras4B, Exposing the Effector Binding Site. Journal of Biological Chemistry, 290, 28887-28900. https://doi.org/10.1074/jbc.M115.664755

[17] Darden, T., York, D. and Pedersen, L. (1993) Particle Mesh Ewald: An N·log (N) Method for Ewald Sums in Large Systems. The Journal of Chemical Physics, 98, 
10089-10092. https://doi.org/10.1063/1.464397

[18] Hess, B., Bekker, H., Berendsen, H.J. and Fraaije, J.G. (1997) LINCS: A Linear Constraint Solver for Molecular Simulations. Journal of Computational Chemistry, 18, 1463-1472.

https://doi.org/10.1002/(SICI)1096-987X(199709)18:12<1463::AID-JCC4>3.0.CO;2$\underline{\mathrm{H}}$

[19] Chen, F., Liu, H., Sun, H., Pan, P., Li, Y., Li, D. and Hou, T. (2016) Assessing the Performance of the MM/PBSA and MM/GBSA Methods. 6. Capability to Predict Protein-Protein Binding Free Energies and Re-Rank Binding Poses Generated by Protein-Protein Docking. Physical Chemistry Chemical Physics, 18, 22129-22139. https://doi.org/10.1039/C6CP03670H

[20] Hou, T., Wang, J., Li, Y. and Wang, W. (2011) Assessing the Performance of the MM/PBSA and MM/GBSA Methods. 1. The Accuracy of Binding Free Energy Calculations Based on Molecular Dynamics Simulations. Journal of Chemical Information and Modeling, 51, 69-82. https://doi.org/10.1021/ci100275a

[21] Sun, H., Li, Y., Tian, S., Xu, L. and Hou, T. (2014) Assessing the Performance of MM/PBSA and MM/GBSA Methods. 4. Accuracies of MM/PBSA and MM/GBSA Methodologies Evaluated by Various Simulation Protocols Using PDBbind Data Set. Physical Chemistry Chemical Physics, 16, 16719-16729. https://doi.org/10.1039/C4CP01388C

[22] Xu, L., Sun, H., Li, Y., Wang, J. and Hou, T. (2013) Assessing the Performance of MM/PBSA and MM/GBSA Methods. 3. The Impact of Force Fields and Ligand Charge Models. The Journal of Physical Chemistry B, 117, 8408-8421. https://doi.org/10.1021/jp404160y

[23] Weiser, J., Shenkin, P.S. and Still, W.C. (1999) Approximate Atomic Surfaces from Linear Combinations of Pairwise Overlaps (LCPO). Journal of Computational Chemistry, 20, 217-230. https://doi.org/10.1002/(SICI)1096-987X(19990130)20:2<217::AID-JCC4>3.0.CO;2$\underline{\mathrm{A}}$

[24] Onufriev, A., Bashford, D. and Case, D.A. (2000) Modification of the Generalized Born Model Suitable for Macromolecules. The Journal of Physical Chemistry B, 104, 3712-3720. https://doi.org/10.1021/jp994072s

[25] Hou, T. and Yu, R. (2007) Molecular Dynamics and Free Energy Studies on the Wild-Type and Double Mutant HIV-1 Protease Complexed with Amprenavir and Two Amprenavir-Related Inhibitors: Mechanism for Binding and Drug Resistance. Journal of Medicinal Chemistry, 50, 1177-1188. https://doi.org/10.1021/jm0609162

[26] Duan, L., Liu, X. and Zhang, J.Z. (2016) Interaction Entropy: A New Paradigm for Highly Efficient and Reliable Computation of Protein-Ligand Binding Free Energy. Journal of the American Chemical Society, 138, 5722-5728. https://doi.org/10.1021/jacs.6b02682

[27] Ni, D., Liu, D., Zhang, J. and Lu, S. (2018) Computational Insights into the Interactions between Calmodulin and the c/nSH2 Domains of p85a Regulatory Subunit of PI3Ka: Implication for PI3Ka Activation by Calmodulin. International Journal of Molecular Sciences, 19, Article No. 151. https://doi.org/10.3390/ijms19010151 